\title{
Relationship between lumbar spinal stenosis and psychosocial factors: a multicenter cross-sectional study (DISTO project)
}

\author{
Miho Sekiguchi $^{1} \cdot$ Koji Yonemoto ${ }^{2} \cdot$ Tatsuyuki Kakuma $^{2} \cdot$ Takuya Nikaido $^{1}$ • \\ Kazuyuki Watanabe $^{1} \cdot$ Kinshi Kato $^{1} \cdot$ Koji Otani $^{1} \cdot$ Shoji Yabuki $^{1}$ • \\ Shin-ichi Kikuchi ${ }^{1} \cdot$ Shin-ichi Konno ${ }^{1}$
}

Received: 22 August 2014/Revised: 4 May 2015/Accepted: 5 May 2015/Published online: 14 May 2015 (C) The Author(s) 2015. This article is published with open access at Springerlink.com

\begin{abstract}
Introduction Lumbar spinal stenosis (LSS) is a lumbar spinal disorder that causes leg symptoms and intermittent claudication. It is reported that the risk factors for low back pain include age, family history, smoking, obesity, workrelated physical load, exercise, and depression. This was a multicenter, cross-sectional survey, and the aim of this study was to investigate the prevalence of LSS by age and the relationships between LSS and psychosocial factors and job satisfaction.

Methods This study enrolled subjects aged 50 years and over from a survey of LSS in 2177 hospitals and general practices nationwide. The clinical characteristics of the LSS and non-LSS groups were compared using the $\chi^{2}$ test, and the multivariate logistic regression analysis was performed to examine associations between exercise, perceived stress, strenuous use of the low back or legs, job satisfaction, and LSS.

Results In total, 18,642 patients (8338 males, 10,267 females) were analyzed. The rate of LSS was $38.3 \%$ and it increased with age. Regular exercise was less common among those in the LSS group than those in the non-LSS group $(p<0.001)$. Satisfaction in all job-related items was less in the LSS group than in the non-LSS group
\end{abstract}

For the DISTO-project group.

Members of the research groups are listed in "Appendix".

Miho Sekiguchi

miho-s@fmu.ac.jp

1 Department of Orthopaedic Surgery, Fukushima Medical University School of Medicine, 1- Hikarigaoka, Fukushima, Fukushima 960-1295, Japan

2 Biostatistics Center, Kurume University, Fukuoka, Japan $(p<0.001)$. The odds for having LSS were higher in subjects having perceived stress and strenuous use of the low back or legs $(p=0.001)$. The odds ratios of heart diseases and hypertension $(p<0.001)$ were higher in the LSS group.

Conclusion This study investigated factors associated with LSS. The prevalence of LSS increased with age. Perceived stress and strenuous use of the low back or legs might be associated with LSS, and job satisfaction was lower with LSS.

Keywords Lumbar spinal stenosis - Psychosocial factors · Job satisfaction - LSS diagnostic support tool

\section{Introduction}

Lumbar spinal stenosis (LSS) is a common spinal disorder that causes various symptoms such as lower extremity pain and/or numbness $[4,8,13,14,23]$. The severity of symptoms such as neurogenic intermittent claudication changes because of posture and activities of daily living. A population-based survey of LSS in Japan [22] used a selfadministered, self-reported history questionnaire for LSS (SSHQ) [9]. The prevalence of LSS over 40 years of age was $5.7 \%$, and the number of LSS patients was estimated to be $3,650,000$ in the Japanese population [22]. Various risk factors such as age, family history, smoking, obesity, exercise, stress, and depression are reported as factors associated with low back pain $[12,17,19,20]$.

Particular attention has been paid to the relationship between psychosocial factors and chronic low back pain, and the therapeutic strategy for low back pain uses various approaches, such as multidisciplinary treatment. Factors associated with LSS have been reported to be age 
(>60 years), comorbidities (diabetes mellitus, urological disorders, osteoarthritis, fracture), and depressive symptoms [22]. In addition, LSS has a negative impact on quality of life [15]. However, few large-scale surveys have been performed to investigate the risk factors of LSS in Japan [22].

The purpose of this study was to investigate the prevalence of LSS by age group in a care-seeking population, as well as to examine whether psychosocial factors and job satisfaction were associated with LSS.

\section{Methods}

\section{Ethics}

This study was approved by the ethics committees of the participating institution.

\section{Study design and population}

The survey of the LSS diagnostic support tool (DISTO) project was a multicenter, cross-sectional study. The DISTO-project survey was conducted under the guidance of the Japanese Society for Spine Surgery and Related Research. The research group consisted of DISTO-project members [16]. The recruitment period was from December 1, 2011 to December 31, 2012. This survey was conducted in 2177 hospitals and general practices nationwide and included 38,577 participants. The participants were patients who visited the participating institutions for any reason including spinal complaints and physicians who worked at the participating institutions.

\section{Data collection}

This study enrolled subjects from the DISTO-project survey who were aged 50 years or over irrespective of their reasons for visiting the institution. The subjects were divided into the LSS group and the non-LSS group. The physicians used the LSS diagnostic support tool (LSSDST) (Table 1) [10], and the subjects were diagnosed with LSS if they had a total score $\geq 7$ (range 0-16) with the LSS-DST. A study to validate the tool that included neurological and imaging findings has been performed [10]. This tool is simple and useful to select a suitable set of subjects in a multicenter study without imaging findings. The LSS-DST consisted of 10 items, with a sensitivity of $92.8 \%$ and specificity of $72 \%$ [10].

\section{Measurements}

The questionnaire was completed by the subjects. The subjects were required to select from the following: (1a) exercise regularly, or (1b) hardly ever exercise; (2a) feel very stressed, or (2b) don't feel very stressed; and (3a) regularly use the low back or legs strenuously (e.g., hard labor, work with same posture, housework for hours) or (3b) don't regularly use the low back or legs strenuously (e.g., light labor-like desk work, do not do much housework). In order to assess the subjects' levels of satisfaction regarding work content, income, human relationships, and workplace environment, the subjects chose a response of (1) satisfaction, (2) slight satisfaction, (3) indifferent, (4) slight dissatisfaction, or (5) dissatisfaction for each item. Physicians asked and recorded if the subjects had the following comorbidities: heart disease, hypertension, hyperlipidemia, or diabetes.
Table 1 Diagnostic support tool for lumbar spinal stenosis

\begin{tabular}{lll}
\hline Item & Score & \\
\hline Age (years) & & \\
$<60$ & 1 & \\
$60-70$ & 2 & No (0) \\
$>70$ & Yes (1) & \\
Diabetes mellitus & & No (0) \\
Symptoms & Yes (3) & No (0) \\
Intermittent claudication & Yes (2) & No (0) \\
Worse when standing for a while & Yes (3) & No (0) \\
Symptoms improve on bending forward & & No (0) \\
Physical examination & Yes (-1) & No (0) \\
Symptoms induced by having patients bend forward & Yes (1) & No (0) \\
Symptoms induced by having patients bend backward & Yes (3) & No (0) \\
Ankle-brachial index (ABI) $\geq 0.9$ & Yes (1) & Yes (-2) \\
Absence or low response of Achilles tendon reflex & & \\
Straight leg raising test positive & & \\
\hline
\end{tabular}

Patients with a total score (range $0-16$ ) of $\geq 7$ are identified as having LSS 


\section{Statistical analysis}

The $\chi^{2}$ test was used to compare the clinical characteristics between the LSS group and the non-LSS group. The Wilcoxon rank sum test was used to compare the job satisfaction of the two groups. In addition, for each factor, the crude odds ratio and the age-sex adjusted odds ratio for having LSS were estimated using logistic regression analysis. $p$ values of less than 0.05 were considered significant. All statistical analyses were conducted using SAS Ver 9.3 (SAS Institute Inc., Cary, NC, USA).

\section{Results}

In total, 18,642 subjects were enrolled as the subjects in the present analysis: 8338 males, 10,267 females, and 37 unknown sex, with most aged 70-79 years. The mean ages were 66 years (range 58-73 years) in the non-LSS group and 73 years (range 68-79 years) in the LSS group.

The number of LSS subjects aged 50 years or over was $7141(38.3 \%)$. The prevalence of LSS was $12.1 \%$ in those aged 50-59 years, $30.4 \%$ in those aged 60-69 years, $50.6 \%$ in those aged 70-79 years, and $60.8 \%$ in those aged 80 years and over, showing an increase with age. The prevalences of LSS in males and females are shown in Fig. 1.

The comparison of subjects' characteristics between the LSS and non-LSS groups is shown in Table 2. The rate of subjects who did regular exercise was lower in the LSS group than in the non-LSS group (42.7 vs $50.1 \%$, $p<0.001)$. The rate of perceived stress was higher in the LSS group than in the non-LSS group (31.6 vs $25.7 \%$, $p<0.001)$. The rate of subjects who used their low back and legs strenuously was higher in the LSS group than in

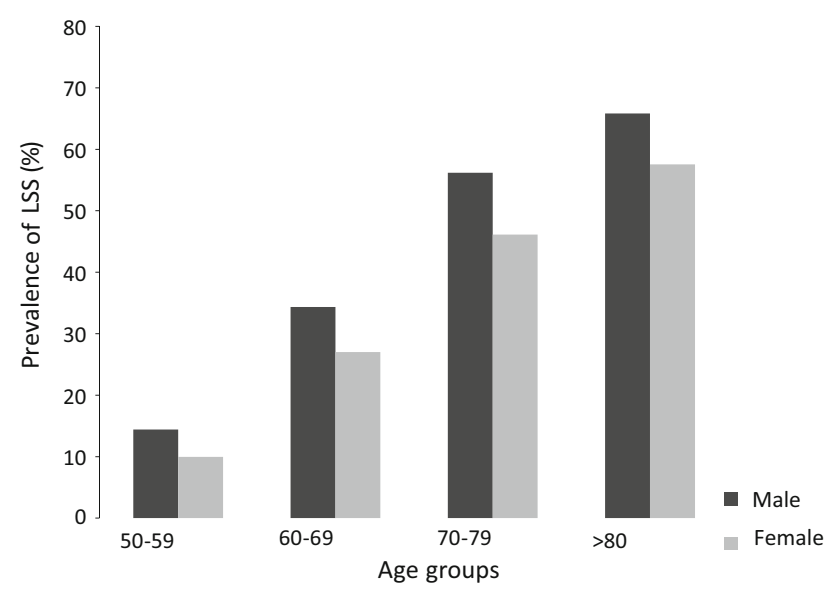

Fig. 1 Male/female prevalence of LSS, respectively: $14.4 \%, 10.0 \%$ (50-59 years), $34.3 \%, 27.0 \%$ (60-69 years), $56.2 \%, 46.1 \%$ (70-79 years), $65.8 \%, 57.5 \%$ ( $>80$ years) the non-LSS group ( 40.3 vs $37.2 \%, p=0.001$ ). The rates of coexisting heart disease ( 9.3 vs $5.4 \%, p<0.01$ ), hypertension ( 35.4 vs $27.7 \%, p<0.01$ ), and hyperlipidemia (13.1 vs $12.1 \%, p=0.02$ ) were higher in the LSS group than in the non-LSS group.

The combined percentage of satisfaction and slight satisfaction was 44.6 and $51.5 \%$ for job content, 34.9 and $39.8 \%$ for income, 50.4 and $55.2 \%$ for human relationships, and 44.3 and $49.3 \%$ for workplace environment in the LSS group and non-LSS group, respectively. Satisfaction for all job-related items was less in the LSS group than in the non-LSS group $(p<0.001)$ (Fig. 2).

The associations of the subjects' characteristics with the presence of LSS are shown in Table 3. The adjusted odds ratios showed that LSS increased with age. Based on the 50-59 years age group, the adjusted odds ratio for subjects in the 70-79 years age group was 7.64. In subjects aged over 80 years, it was 11.84 . The adjusted odds ratio of males for having LSS was 1.46. The odds for having LSS

Table 2 Characteristics of subjects with and without lumbar spinal stenosis (LSS)

\begin{tabular}{|c|c|c|c|}
\hline & $\%(n)$ & & \\
\hline & $\begin{array}{l}\operatorname{LSS}(-) \\
(n=11,501)\end{array}$ & $\begin{array}{l}\operatorname{LSS}(+) \\
(n=7141)\end{array}$ & $p$ \\
\hline Age & & & $<0.001$ \\
\hline $50-59$ & $28.2(3245)$ & $6.3(447)$ & \\
\hline $60-69$ & $34.9(4012)$ & $24.5(1751)$ & \\
\hline $70-79$ & $27.0(3101)$ & 44.4 (3171) & \\
\hline $80-$ & 9.9 (1143) & $24.8(1772)$ & \\
\hline Sex & & & $<0.001$ \\
\hline Male & $42.4(4861)$ & 48.8 (3477) & \\
\hline Female & $57.6(6617)$ & $51.2(3650)$ & \\
\hline Exercise habit & & & $<0.001$ \\
\hline Yes & $50.1(5514)$ & 42.7 (2948) & \\
\hline No & 49.9 (5489) & $57.3(3958)$ & \\
\hline Perceived stress & & & $<0.001$ \\
\hline Yes & $25.7(2822)$ & $31.6(2178)$ & \\
\hline No & $74.3(8145)$ & $68.4(4714)$ & \\
\hline $\begin{array}{l}\text { Strenuous use of low back or } \\
\text { legs }\end{array}$ & & & 0.001 \\
\hline Yes & $37.2(3550)$ & $40.3(2301)$ & \\
\hline No & $62.8(5989)$ & 59.7 (3404) & \\
\hline Number of comorbidities & & & $<0.001$ \\
\hline 0 & $59.3(6819)$ & 52.9 (3776) & \\
\hline $1 \leq$ & $40.7(4682)$ & $47.1(3365)$ & \\
\hline Comorbidities & & & \\
\hline Heart disease & $5.4(615)$ & $9.3(662)$ & $<0.001$ \\
\hline Hypertension & 27.7 (3190) & 35.4 (2529) & $<0.001$ \\
\hline Hyperlipidemia & $12.1(1390)$ & $13.3(946)$ & 0.02 \\
\hline Diabetes & 12.5 & $11.9(846)$ & 0.2 \\
\hline
\end{tabular}


Fig. 2 Satisfaction with the contents of work, income, human relationships, and the workplace environment. The satisfaction for all job-related items is less in the LSS group than in the non-LSS group $(p<0.001)$
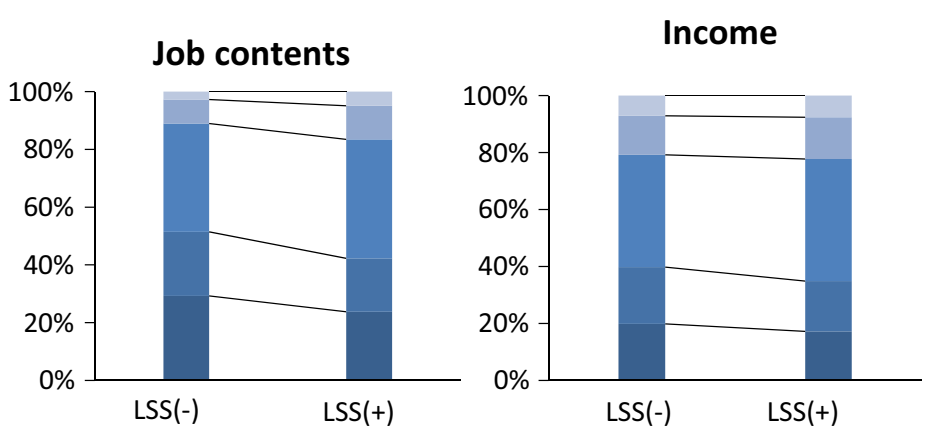

Human relations

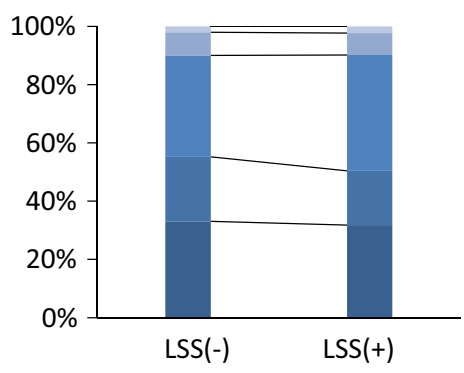

Workplace environment

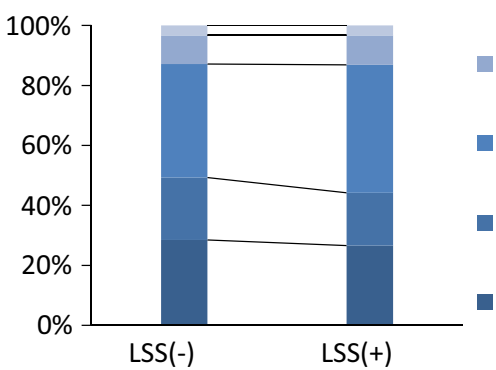

dissatisfaction

slight dissatisfaction

- indifferent

slight

satisfaction

satisfaction
Table 3 Factors related to LSS on multivariate logistic regression analysis

\begin{tabular}{|c|c|c|c|}
\hline & Adjusted OR & $95 \% \mathrm{CI}$ & $p$ value \\
\hline \multicolumn{4}{|c|}{ Age (vs. 50-59 years) } \\
\hline $60-69$ & 3.21 & $(2.87-3.60)$ & $<0.001$ \\
\hline $70-79$ & 7.64 & $(6.83-8.54)$ & $<0.001$ \\
\hline$\geq 80$ & 11.84 & $(10.45-13.41)$ & $<0.001$ \\
\hline \multicolumn{4}{|l|}{ Sex (vs. female) } \\
\hline Male & 1.46 & $(1.37-1.56)$ & $<0.001$ \\
\hline \multicolumn{4}{|c|}{ Exercise habit (vs. yes) } \\
\hline No & 1.46 & $(1.36-1.55)$ & $<0.001$ \\
\hline \multicolumn{4}{|c|}{ Perceived stress (vs. no) } \\
\hline Yes & 1.69 & $(1.57-1.82)$ & $<0.001$ \\
\hline \multicolumn{4}{|c|}{ Strenuous use of low back or legs (vs. no) } \\
\hline Yes & 1.41 & $(1.31-1.52)$ & $<0.001$ \\
\hline \multicolumn{4}{|c|}{ Number of comorbidities (vs. none) } \\
\hline$\geq 1$ & 1.06 & $(0.99-1.13)$ & 0.08 \\
\hline \multicolumn{4}{|l|}{ Comorbidities } \\
\hline Heart disease & 1.28 & $(1.14-1.45)$ & $<0.001$ \\
\hline Hypertension & 1.16 & $(1.08-1.24)$ & $<0.001$ \\
\hline Hyperlipidemia & 1.06 & $(0.97-1.17)$ & 0.22 \\
\hline Diabetes & 0.82 & $(0.75-0.90)$ & $<0.001$ \\
\hline
\end{tabular}

ORs for age were adjusted by sex, and ORs for sex were adjusted by age

ORs for other items were adjusted by age and sex

were higher in subjects who lacked regular exercise $(\mathrm{OR}=1.46)$, who perceived stress $(\mathrm{OR}=1.69)$, and who were involved in hard labor $(\mathrm{OR}=1.41)$. Concerning the number of comorbidities, the crude odds ratio was 1.3 in subjects with 1 or more comorbidities, and the adjusted odds ratio for this association was not significant $(\mathrm{OR}=1.06)$. In regard to individual comorbidities, heart disease $(\mathrm{OR}=1.28)$ and hypertension $(\mathrm{OR}=1.16)$ showed increased adjusted odds ratios for having LSS. In contrast, the adjusted odds ratio for LSS was 0.82 in subjects with diabetes.

\section{Discussion}

This study found that the prevalence of LSS was $38.3 \%$ and increased with age. Two-thirds of the LSS subjects were aged 70 years or over. Furthermore, the prevalence of LSS was greater in male subjects than in female subjects. According to a general population survey, there was no difference in LSS by sex [22], while other results have found more male than female subjects with LSS [21]. The distribution of a hospital-based survey might be different from that of the general population, and the disability, severity, or progress of LSS might differ between the sexes.

The rates of lacking regular exercise and strenuous use of the low back or legs were higher in the LSS group than in the non-LSS group in the present study. The odds ratio for strenuous use of the low back or legs for having LSS was 1.41 . In the present study, neither the subjects' occupations nor load intensities were evaluated; however, the survey question regarding strenuous use of the low back or legs included an explanation of what constitutes strenuous 
use. Strenuous use included physical labor, maintaining a posture for a prolonged period, and extensive housekeeping, and non-strenuous use included office work, light work, brief housekeeping, little work, or little housekeeping. It is reported that LSS is more likely in males performing heavy manual labor and females doing housekeeping [11]. In addition, low back pain is associated with a physically heavy workload at work and lower physical activity during leisure time [3]. In contrast, no difference in exercise habits was found between LSS and non-LSS subjects in a population-based study [21]. Lack of regular exercise seems to be similar to less physical activity. It is still not clear whether doing less physical activity is the result of the severity of symptoms or vice versa. The present result suggests an association between LSS and both strenuous use of the low back or legs and a lack of exercise.

The rate of perceived stress was significantly higher in the LSS group (31.6\%) than in the non-LSS group (25.7\%). In addition, satisfaction levels for job content, income, human relationships, and workplace environment were lower in the LSS group than in the non-LSS group. It has been reported that there was no difference in perceived stress using the Japanese Perceived Stress Scale, but the odds of severe depressive symptoms were significantly higher in LSS subjects than in non-LSS subjects in a population-based study [22]. Another study reported that people dissatisfied with work are more likely to report low back pain (LPB) [17]. In addition, Takahashi et al. [19] investigated subjects with LBP, and though they did not evaluate the cause of LBP, they found that people with a high level of disability, but only mild LBP, were more depressed and less satisfied with their job content, income, working conditions, and relationships with coworkers. In a previous study, LSS subjects with psychiatric problems showed improvement in their psychiatric problems 1 year after posterior decompression surgery [5]. In contrast, LSS subjects, who did not show improvement in their psychiatric problems after surgical treatment, had poor outcomes and lower satisfaction with treatment [5]. The present result suggests that perceived stress might be associated with LSS, and LSS patients should be checked for the presence of relevant psychosocial factors.

In the present study, there were increases in the odds for having LSS among those having heart diseases and hypertension, while there was a decrease in the odds among those with diabetes mellitus compared with no comorbidities. There are some reports that states hypertension is associated with LSS [2, 21]. In contrast, other studies found that hypertension is not associated with $\operatorname{LSS}[1,22]$, and heart disease has no reported relationship with LSS. Furthermore, diabetes mellitus has already been reported to be associated with LSS [22]. Non-elderly patients with LSS were more likely to have diabetes mellitus, compared with the general population, while there was no difference in the proportion of patients with diabetes mellitus between LSS subjects and the general elderly population [21]. Diabetes mellitus increases the risk of aortic calcification, and aortic calcification correlates not only with hypertension and heart diseases, but also degeneration of the intervertebral disks and spine [6, 7, 18]. These comorbidities might be associated with LSS, and the kinds of comorbidities might not be different according to the data collection setting.

This study has some limitations. First, data were collected from both orthopedic and non-orthopedic departments in hospitals and general practices. Therefore, in the primary care setting, undiagnosed LSS subjects receiving treatment unrelated to LSS would also be included. In addition, comorbidities were self-reported; therefore, some subjects might have an unknown comorbidity not under treatment. Furthermore, the distribution of patients varied according to where the data were collected, especially with respect to the individual reason for visiting a medical institution.

Second, subjects with severe symptoms may have been included because the subjects were taken from hospitals and the care-seeking population may potentially have more severe LSS than the general population. However, disability in daily living and the severity of LSS were not examined in each patient. There was no investigation of the amount of stress, the degree of loading, the amount of variation within each item, or the relationship of stress to the degree of LSS.

Third, the specificity of $72 \%$ of the LSS-screening tool has to be mentioned as a limitation of the study, as it implies that $28 \%$ of the diagnosed LSS patients were potentially false positives.

Fourth, the absence of diabetes mellitus added one point for the LSS-DST in order to distinguish peripheral neuropathy due to diabetes mellitus. Therefore, the rate of diabetes mellitus might be affected among the subjects who were chosen using the LSS-DST.

Finally, this was a cross-sectional study. Therefore, there was no mention of the causal relationships between strenuous use of the low back or legs and exercise and LSS. In addition, although psychosocial factors are considered to be associated with treatment effectiveness, the relationship between these risk factors and LSS treatment effectiveness is yet to be fully investigated. An evaluation of psychosocial factors is important to indicate which LSS treatment is suitable. Therefore, in order to predict and intervene in the cases of symptomatic LSS with risk factors for LSS, further longitudinal studies are needed.

In conclusion, the prevalence of LSS increased with age. Lack of regular exercise, perceived stress, and strenuous use of the low back or legs might be associated with LSS. In addition, job satisfaction was lower with LSS. 
Acknowledgments This project was supported for data collection and analysis from the Japanese Society for Spine Surgical and Related Research.

\section{Conflict of interest None.}

Open Access This article is distributed under the terms of the Creative Commons Attribution 4.0 International License (http:// creativecommons.org/licenses/by/4.0/), which permits unrestricted use, distribution, and reproduction in any medium, provided you give appropriate credit to the original author(s) and the source, provide a link to the Creative Commons license, and indicate if changes were made.

\section{Appendix: Lumbar Spinal Stenosis Diagnosis Support Tool (DISOTO)-project group}

Chairman: Shin-ichi Kikuchi (Department of Orthopaedic Surgery, Fukushima Medical University School of Medicine).

Research members: Shin-ichi Kikuchi, Shin-ichi Konno, Miho Sekiguchi (Department of Orthopaedic Surgery, Fukushima Medical University School of Medicine), Kazuhisa Takahashi, Seiji Ohtori (Department of Orthopaedic Surgery, Graduate School of Medicine, Chiba University), Tatsuyuki Kakuma, Koji Yonemoto (Department of Bio-statistics, Kurume University School of Medicine), Kazuo Yonenobu (Department of Orthopaedic Surgery, Osaka Minami Medical Center), Keisuke Takahashi, Hideki Iizuka (Department of Orthopaedic Surgery, Saitama Medical University, Faculte of Medicine), Toshihiko Taguchi, Tukasa Kanchiku (Department of Orthopaedic Surgery Yamguchi University Faculty of Medicine and Health Sciences), Katsushi Takeshita, Nobuhiro Hara (Department of Orthopaedic Surgery, The University of Tokyo Faculty of Medicine), Toshikazu Tani, Ryuichi Takemasa (Department of Orthopaedic Surgery, Kochi Medical School), Kazuhiro Chiba (Department of Orthopaedic Surgery, Kitasato University Kitasato Institute Hospital), Naobumi Hosogane (Department of Orthopaedic Surgery, Keio University School of Medicein), Kensei Nagata, Kimiaki Sato (Department of Orthopaedic Surgery, Kurume University School of Medicien), Yutaka Nohara, Hiroshi Taneichi (Department of Orthopaedic Surgery, Dokkyo Medical University School of Medicine), Yuichi Hoshino, Atsushi Seichi (Department of Orthopaedic Surgery, Jichi Medicau University), Toshihiko Yamashita, Tsuneo Takebayashi (Department of Orthopaedic Surgery, Sapporo Medical University School of Medicine), Munehito Yoshida, Hiroshi Yamada (Department of Orthopaedic Surgery, Wakayama Medical University).

Research associate members: Takuya Nikaido, Kazuyuki Watanabe, Kinshi Kato ((Department of
Orthopaedic Surgery, Fukushima Medical University School of Medicine).

\section{References}

1. Abbas J, Hamoud K, May H et al (2013) Socioeconomic and physical characteristics of individuals with degenerative lumbar spinal stenosis. Spine 38:E554-E561

2. Battie MC, Jones CA, Schopflocher DP et al (2012) Health-related quality of life and comorbidities associated with lumbar spinal stenosis. Spine J 12:189-195

3. Bjorch-van Dijken C, Fjellman-Wiklund A, Hildngsson C (2008) Loa back pain, lifestyle factors and physical activity: a population based-study. J Rehabil Med 40:864-869

4. Kalichman L, Cole R, Kim DH et al (2009) Spinal stenosis prevalence and association with symptoms: the Framingham study. Spine J 9:545-550

5. Kato K, Otani K, NIkaido T et al (2014) Relation between surgical treatment outeoms and psychiatric problems in lumbar spinal stenosis. A prospective cohort study. Rinsyoseikei 49:123-129 (Japanese)

6. Kauppila LI (2009) Atherosclerosis and disc degeneration/low back pain-a systematic review. Eur J Vasc Endovasc Surg 37:661-670

7. Kauppila LI, Penttila A, Karhunen PJ et al (1994) Lumbar dis degeneration and stherosclerosis of the abdominal aorta 19:923-929

8. Kim HJ, Suh BG, Lee DB et al (2013) The influence of pain sensitibity on the symptom severity in patients with lumbar spinal stenosis. Pain Physician 16:135-144

9. Konno S, Kikuchi S, Tanaka Y et al (2007) A diagnostic support tool for lumbar spinal stenosis: a self-administered, self-reported history questionnaire. BMC Muscloskelet Disord 8(102):1-9

10. Konno S, Hayashino Y, Fukuhara S et al (2007) Development of a clinical diagnosis support tool to identify patients with lumbar spinal stenosis. Eur Spine J 16:1951-1957

11. Lotan R, Oron A, Anekstein Y et al (2008) Lumbar stenosis and systemic disease: is there any relebanve? J Spine Disord Tech 21:245-247

12. Ono R, Yamazaki S, Takegami M et al (2012) Gender difference in association between low back pain and metabolic syndrome. Spine 37:1130-1137

13. Otani K, Kikuchi S, Konno S et al (2008) Epidemiologic study of musculoskeletal disorders. The minami-aizu study. Part 1: Crosssectional study of the incidence of lumbar spinal canal stenosis and its relation to low-back- pain-related QOL. Rinsyoseikei 43:789-796 (Japanese)

14. Otani K, Kikuchi S, Konno S et al (2008) Epidemiologic study of musculoskeletal disorders. The Minami-Aizu study. Part 2: Cross-sectional study of the incidence of lumbar spinal canal stenosis and its relation to health-related QOL (HR-QOL). Rinsyoseikei 43:883-888 (Japanese)

15. Otani K, Kikuchi S, Yabuki S et al (2013) Lumbar spinal stenosis has a negative impact on quality of life compared with other comorbidities: an epidemiological cross-sectional study of 1862 community-dwelling indivisuals. Sci Word J 2013:1-9

16. Ohtori S, Sekiguchi M, Yonemoto K et al (2014) Awareness and use of diagnostic support tools for lumbar spinal stenosis in Japan. J Orthop Sci 19:412-417

17. Papageorgiou AC, Macfarlane GJ, Thomas E et al (1997) Psychosocial factors in the workplace-do they predict nes episodes 
of low back pain? Evidence from the south Manchester back pain study. Spine 22:1137-1142

18. Sakellaridis N (2006) The influence of diabetes mellitus on lumbar intervertebral disk herniation. Surg Neurol 66:152-154

19. Takahashi N, Kikuchi S, Konno S et al (2006) Discrepancy between disability and the severity of low back pain: demographic, pcyshologic, and employment-related factors. Spine 31:931-939

20. Tomkins-Lane CC, Holz SC, Yamakawa KS et al (2012) Predictors of walking performance and walking capacity in people with lumbar spinals stenosism low back pain and asymptomatic controls. Arch Phys Med Rehabil 93:647-653

21. Uesugi K, Sekiguchi M, Kikuchi S et al (2013) Relationship between lumbar spinal stenosis and lifestyle-related disorders: a cross-sectional multi-center observational study. Spine 38:E540 E545

22. Yabuki S, Fukumori N, Takegami M et al (2013) Prevalence of lumbar spinal stenosis, using the diagnostic support tool, and correlated factors in Japan: a population-based study. J Orthop Sci 18:893-900

23. Zeifang F, Schiltenwolf M, Abel R et al (2008) Gait analysis does not prelate with clinical and MR imaging parameters in patients with symptomatic lumbar spinal stenosis. BMC Muscloskelet Disord 20:1-8 